
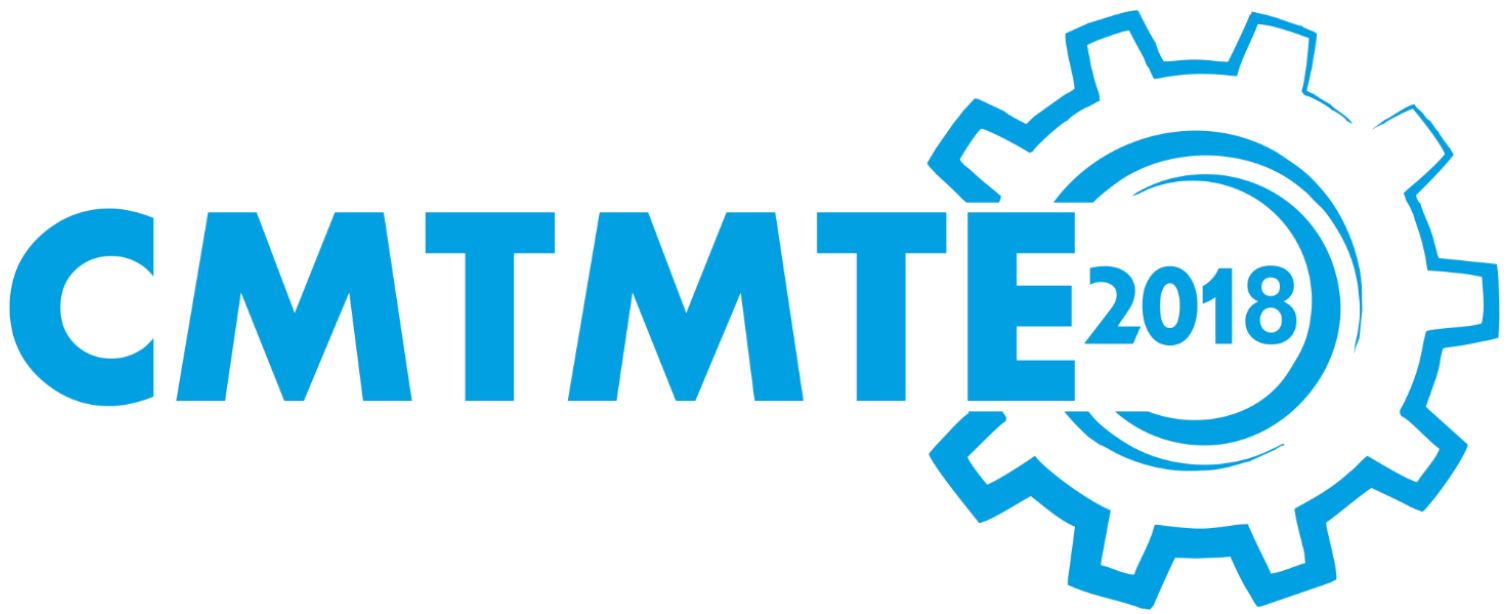

\title{
PREFACE
}

The International Conference on Modern Trends in Manufacturing Technologies and Equipment 2018 (ICMTME 2018) was held in Sevastopol, Russia from 10 to 14 September 2018 and was organized by the Sevastopol State University, National University of Science and Technology «MISIS», Polzunov Altai State Technical University, Inlink Ltd. and International Union of Machine Builders.

ICMTMTE 2018 was organized to provide scientists and industry experts with the opportunity to summarize the latest developments in manufacturing technologies, materials science, manufacturing equipment and tools, to discuss future developments in the new technique and technologies, to build up the network and partnerships for the future collaboration.

The conference was supported financially by the Russian Foundation for Basic Research (grant № 18-08-20074).

The conferees represented different countries - Russia, Ukraine, Turkey, Germany, China and Algeria. The proceedings of ICMTMTE 2018 consists of 320 selected papers, which were presented orally at the conference or via poster sessions.

We express our deepest gratitude to all members of the scientific committee, invited speakers, reviewers, sponsors, participants and members of the ICMTMTE 2018 for their valuable contribution to the successful organization of ICMTMTE 2018. We also would like to express our special thanks to all staff from EDP Sciences for their great support to ICMTMTE 2018.

With best wishes,

Prof. Sergey Bratan, General Chair of ICMTMTE 2018

Dr. Stanislav Roshchupkin, Secretary of ICMTMTE 2018 


\section{Conference Chair}

Prof. Sergey Bratan (Sevastopol State University, Sevastopol, RUS)

\section{Conference Co-Chair}

Prof. Sergey Gorbatyuk (National University of Science and Technology «MISIS», Moscow, RUS)

Prof. Alexander Glezer (National University of Science and Technology «MISIS», Moscow, RUS)

Prof. Sergey Leonov (Polzunov Altai State Technical University, Barnaul, RUS)

\section{International Program Committee}

Prof. U. Abdulgazis (Crimean. Eng. and Ped. Un., RUS)

Prof. T. Duyun (Belgorod St. Technol. Un., RUS)

Prof. A. Mikhaylov (Donetsk National Tech. Un., UKR)

Prof. G. Bahadirov (IMSS named after Urazbaeva, UZB)

Prof. A. Buchacz (Silesian Technical University, POL)

Prof. N. Danijarov (Karaganda St. Tech. Un., KAZ)

Prof. Y. Novoselov (Sevastopol State Un., RUS)

Prof. A. Kharchenko (Sevastopol State Un., RUS)

Prof. A. Bohonskiy (Sevastopol State Un., RUS)

Prof. N. Pokintelitsa (Sevastopol State Un., RUS)

Prof. R. Giniavichus (Vilnius Gediminas Tech. Un., LTU)

Assoc. Prof. A. Giutuni (Tunis Tech. University, TUN)

Prof. M. Heyfetc (Nat. Academy of Sc. of Belarus, BLR)

Prof. A. Handozhko (Bryansk St. Tech. University, RUS)

Assoc. Prof. G-J. Jasper (Plymouth University, GBR)

Prof. R. Kasper (OVG University Magdeburg, DEU)

Prof. S. Khristoforian (St. Eng.Un. of Armenia, ARM)

Prof. G. Barsukov (Orel State University, RUS)

Prof. O. Pruteanu (Tech. Un. “Gh. Asachi”, ROU)

Prof. M. Radovanovic (Tech. Un. of Nis, SRB)

Prof. A. Toca (Technical University Moldova, MDA)

Assos. prof. A. Srivastava (Shamb. In. of Eng. and Technol. IND)

Assoc. Prof. K. Monkova (Tech. University Kosice, SVK)

Prof. A. Popov (Omsk State Technical University, RUS)

Prof. Z. Sharifov (Azerbaijan Technical. University, AZE)

Prof. A. Kozlov (Lipetsk State Tech. Un. RUS)

Prof. A. Suslov (Moscow State Industrial University, RUS)

Prof. V. Gusev (Donetsk National Tech. Un., UKR)

Prof. F. Yakubov (Crimean. Eng. and Ped. Un., RUS)

Prof. A. Malikov (Tula State University, RUS)

Prof. V. Nosenko (Volgograd State Tech. Un. RUS)

Prof. B. Yazyev (Don State Technical University, RUS)

Prof. A. Korotkov (Kuzbas State Tech. Un., RUS)

Assoc. Prof. S. Litvinov (Don State Technical University, RUS)

Assoc. Prof. P. Novikov (Sevastopol State Un., RUS)

Assoc. Prof. V. Bogutskiy (Sevastopol State Un., RUS)

Assoc. Prof. A. Tarakhovskiy (Sevastopol State Un., RUS)

Assoc. Prof. E. Levchenko (Sevastopol State Un., RUS)

Assoc. Prof.A. Kolesov (Sevastopol State Un., RUS)

M.Sc. M. Pyankovskaya (Sevastopol State Un., RUS)

\section{Conference Secretary}

Assoc. Prof. S. Roshchupkin (Sevastopol State Un., RUS) 

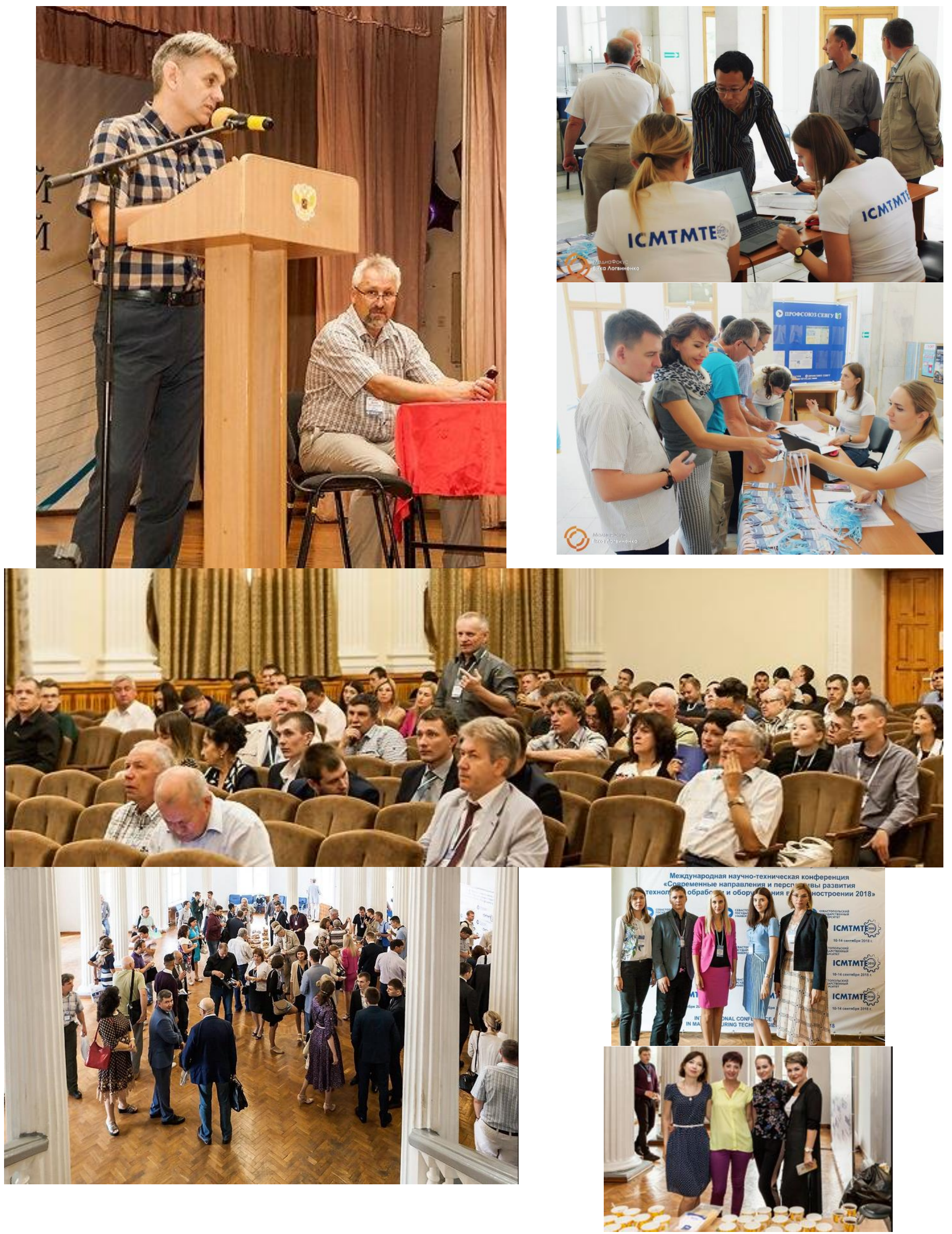

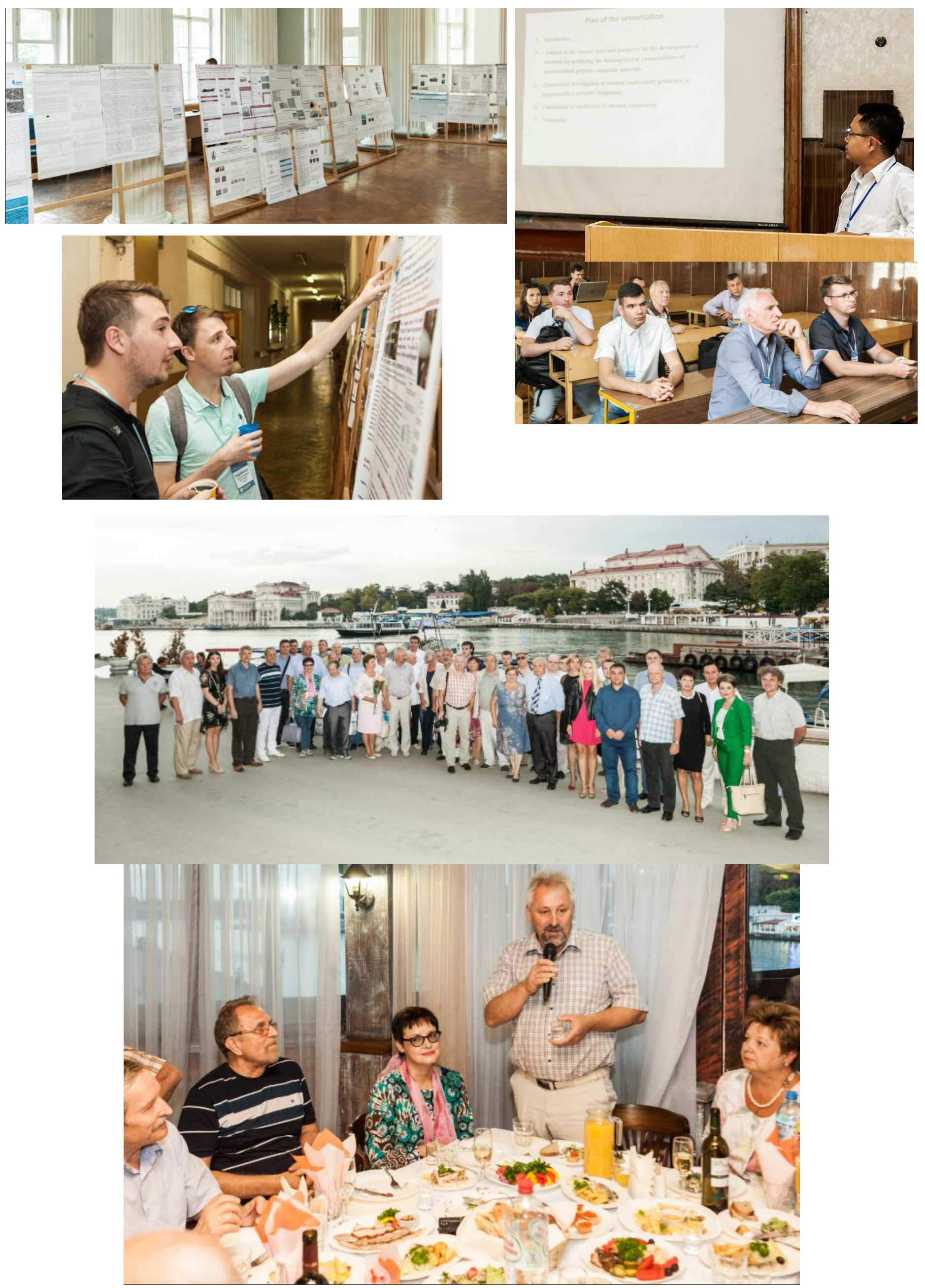